\title{
COMMENT
}

${ }^{1}$ Division of Gastroenterology, Hepatology and Nutrition, The Ohio State University College of Medicine, Columbus, $\mathrm{OH}$, USA.

${ }^{2}$ The Ohio State University Comprehensive Cancer Center, Columbus, OH, USA

${ }^{3}$ Harvard T.H. Chan School of Public Health, Boston, MA, USA.

${ }^{4}$ Division of Gastroenterology and Hepatology, NYU Langone Health, New York, NY, USA.

${ }^{5}$ Clinical Research Division, Fred Hutchinson Cancer Research Center, Seattle, WA, USA.

${ }^{6}$ Division of Gastroenterology, University of Washington School of Medicine, Seattle, WA, USA.

${ }^{7}$ Vatche and Tamar Manoukian Division of Digestive Diseases, Department of Medicine, David Geffen School of Medicine at UCLA, Los Angeles, CA, USA.

${ }^{8}$ Jonsson Comprehensive Cancer Center, University of California Los Angeles, Los Angeles, CA, USA.

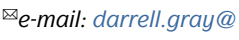
osumc.edu

\section{COVID-19 and the other pandemic: populations made vulnerable by systemic inequity}

Darrell M. Gray II ${ }^{1,2 \bowtie}$, Adjoa Anyane-Yeboa ${ }^{3}$, Sophie Balzora ${ }^{4}$, Rachel B. Issaka ${ }^{5,6}$ and Folasade P. May ${ }^{7,8}$

Greater than the coronavirus disease 2019 (COVID-19) crisis, systemic inequity in social determinants of health is the pandemic that has long fostered vulnerability to disease and poor health outcomes in the USA. Our response has major implications for the health of our nations.

\begin{abstract}
"When you're in the middle of a crisis, like we are now with the coronavirus, it really does...ultimately shine a very bright light on some of the real weaknesses and foibles in our society." - Anthony Fauci, Director of the National Institute of Allergy \& Infectious Diseases, White House Coronavirus Task Force.
\end{abstract}

The coronavirus disease 2019 (COVID-19) pandemic caused by severe acute respiratory syndrome coronavirus 2 (SARS-CoV-2) infection has removed the veil on health inequities that, for some with the luxury to ignore them, have been hiding in plain sight. For others, particularly the most vulnerable among us, these inequities have been tangible and the plague of their lived experiences. In particular, African Americans, Latino individuals, and Native Americans in the USA have experienced a disproportionate burden of COVID-19-related infections and deaths ${ }^{1}$. Although much attention has focused on the high burden of chronic disease among these populations, which predisposes them to poor outcomes if they acquire COVID-19, there is less recognition of the non-medical health-related social needs and social determinants of health $(\mathrm{SDOH})^{2}$ that represent the root causes of such health disparities.

Vulnerability, and specifically vulnerability in health, is not equally distributed. It tends to be concentrated in areas where $\mathrm{SDOH}$ (for example, limited educational attainment, low socioeconomic status, unemployment, discrimination and structural racism) and the non-medical health-related social needs that result from these SDOH contribute to downstream adverse health outcomes (such as cancer, cardiovascular disease and obesity). With poor access to and low utilization of high-quality health care, those who are vulnerable are also medically underserved. Thus, the observed racial and ethnic disparities in COVID-19 cases, hospitalizations and deaths in the USA ${ }^{1}$ is a symptom of upstream systemic inequity - the 'other pandemic' — and interwoven with individual biology, health behaviours and environments (BOX 1).

\section{A baseline of disadvantage}

Vulnerable populations are heterogenous in composition (for example, those living with disability, people living in poverty and racial, ethnic, sexual and gender minorities), and the intersection of these identities within an individual or group can further compound their baseline disadvantage. African Americans, for example, have the highest mortality from colorectal cancer (CRC), the second leading cause of cancer-related death in the USA, and are diagnosed at younger ages (average 64 years) compared with white individuals (average 68 years) ${ }^{3}$. African Americans who are poor, uninsured and/or are geographically isolated in the USA lack access to and are less likely to receive life-saving CRC screening tests; they are also less likely to receive treatment for advanced disease when diagnosed ${ }^{4}$.

Likewise, Latino individuals in the USA are disproportionately affected by many medical conditions that are influenced heavily by SDOH. Individuals of Hispanic or Latino ancestry in the USA have the highest incidence of nonalcoholic fatty liver disease (NAFLD) ${ }^{5}$. Given that Latino individuals represent one of the largest minority groups in the USA and that NAFLD has emerged as a common precursor to hepatocellular carcinoma, one of few gastrointestinal malignancies with increasing national incidence, understanding and addressing the health factors and $\mathrm{SDOH}$ that predispose this population to NAFLD is also critically important.

Importantly, the disparities seen in health and health care are not solely the result of personal health and lifestyle choices - they are also iatrogenic. For example, implicit biases - unconscious stereotypes shaped by learned associations that inform understanding and decision-making - can negatively influence 
Box 1 Social determinants of health and health outcomes during COVID-19

Upstream social determinants of health $(\mathrm{SDOH})$ are the root causes of health disparities at the population level. Midstream SDOH are the result of upstream determinants and mediate the effects of upstream $\mathrm{SDOH}$ on downstream health outcomes. Downstream health outcomes describe the health-related end points that result from upstream and midstream SDOH. Examples of each determinant and possible health outcomes are listed.

Upstream determinants
- Racism
- Discrimination
- Social policies
- Poverty
Midstream determinants
- Educational attainment
- Employment status
- Safety of housing and quality
of living conditions
- Stability of income
- Food security and quality
of diet
- Exposure to toxins
- Medical mistrust

- Access to high-quality health care - Access to COVID-19 testing and care

- Ability to physically distance during the COVID-19 pandemic

- Access to masks during the COVID-19 pandemic

Downstream health outcomes

- Heart disease

- Asthma and lung disease

- Obesity or overweight

- Type 2 diabetes mellitus

- Cancer

- COVID-19 cases, hospitalizations and deaths

COVID-19, coronavirus disease 2019. patient-provider communication and result in worse health-care outcomes. Biases can also adversely influence decisions about liver transplantation candidacy, treatment recommendations for inflammatory bowel disease and cancer treatment ${ }^{6}$. Such biases compound the challenges that vulnerable populations already face through $\mathrm{SDOH}$ and non-medical health-related social needs.

\section{Inequity under the microscope of COVID-19}

Amidst the COVID-19 pandemic, such systemic inequities and implicit biases have become more visible. A study published in 2020 involving seven US states and 67,610 patients who screened positive for one or more symptoms of COVID-19 found that African Americans were less likely to receive a confirmatory test than their white counterparts presenting with similar symptoms ${ }^{7}$. Limited access to primary care services and COVID-19 testing centres present additional barriers to appropriate diagnosis and treatment. For the Native American community in particular, a low number of intensive care hospital beds in the Indian Health Services has limited the ability to provide adequate care for individuals with COVID-19 on tribal reservations ${ }^{8}$. Furthermore, the over-representation of racial and ethnic minorities among essential workers and those living in poor and overcrowded housing conditions makes physical distancing and sheltering-in-place, public health interventions intended to minimize risk of SARS-CoV-2 transmission, challenging for some and impossible for others ${ }^{9}$.

Through the lens of gastroenterology and hepatology practice during the COVID-19 response, our patients are experiencing similar inequities. The medically underserved - those already underutilizing CRC screening and more likely to die from $\mathrm{CRC}^{4}$ - are facing even greater hurdles to timely CRC screening and diagnosis during the COVID-19 pandemic. The postponement of non-urgent procedures, including colonoscopies, will likely widen the gap in late-stage CRC diagnoses and CRC-related deaths ${ }^{10}$. For individuals with chronic liver conditions, routine provider visitation and adherence to dietary modifications are standard of care; however, access to health-care services and affordable, healthy food options is further impaired during the pandemic. Thus, the interaction between these SDOH and COVID-19 could increase the risk of progression of other disease states among the medically underserved.

\section{The path to health equity}

As we navigate COVID-19 and the other pandemic of systemic inequity, we must not only address the downstream symptoms of the disproportionate number of deaths from COVID-19 and chronic diseases within communities of colour. It is imperative that we simultaneously tackle the midstream and upstream causes (BOX 1). To do so, implementation of at least three key strategies is necessary in the USA. These recommendations can be considered in other racially and ethnically heterogeneous settings worldwide.

First, federal, state and local support for health disparities research and workforce diversity in health care and science must increase. Immediate needs include the collection and reporting of accurate and detailed national data on race, ethnicity and SDOH. Furthermore, researchers should be challenged to stretch beyond investigating implications of SDOH and health disparities to the discovery and implementation of interventions to reduce inequities. In the long-term, we must bolster financial resources allocated to pipeline programmes and minority-serving institutions, substantially increasing funding for the National Institute on Minority Health and Health Disparities (one of the lowest-funded NIH institutes in the USA), and mandating implicit bias training and mitigation for grant review committee members.

Second, racism must be declared a public health crisis and resources need to be deployed to mitigate it. There have been examples of counties and/or cities across the USA that have done so. In May 2020, Franklin County Public Health (Ohio, USA) declared racism a public health crisis and a Center for Public Innovation was created to address it. This centre aims to reduce inequities and improve life expectancy and quality of life among residents. Yet, there remains a need for a national legislative priority with long-term investment aiming to strengthen the public health infrastructure and its ability to respond efficiently and effectively to local and national public health crises, dismantle discriminatory policies and practices, and foster healthier communities, starting with the most vulnerable. Especially now, in the wake of deadly violence towards African Americans like George Floyd in the USA and the associated global protests that have ensued beginning at the end of May 2020, systemic racism must be confronted and dismantled.

Third, those most proximal to the inequity must be given voice to propose and implement solutions. Meaningful partnerships between public and private sector stakeholders, civic organizations and local grassroots efforts will build social capital, foster resilience, 
cultivate political will and effectively challenge discriminatory policies, practices and norms that disproportionately affect vulnerable populations. National medical societies and organizations must also be invested in this response. All have a mission to improve and promote health, which cannot be accomplished without addressing the needs of the medically underserved.

These calls are meant to achieve health equity in which everyone has a fair and just opportunity to attain their highest level of health possible. Achieving this goal requires greater attention to medically underserved populations who are made vulnerable by virtue of their SDOH and non-medical health-related social needs. How we respond in times of crisis, when a bright light accentuates systemic inequities and opportunities to eliminate them, is telling of the road ahead.

1. Hooper, M. W., Napoles, A. M. \& Perez-Stable, E. J. COVID-19 and racial/ethnic disparities. JAMA https://doi.org/10.1001/jama. 2020.8598 (2020)

2. National Academies of Sciences, Engineering, and Medicine. Investing in Interventions That Address Non-Medical, Health-Related Social
Needs: Proceedings of a Workshop. https://doi.org/10.17226/25544 (NAP, 2019).

3. Siegel, R. L. et al. Colorectal cancer statistics, 2020. CA Cancer J. Clin. https://doi.org/10.3322/caac.21601 (2020).

4. Carethers, J. M. \& Doubeni, C. A. Causes of socioeconomic disparities in colorectal cancer and intervention framework and strategies. Gastroenterology 158, 354-367 (2020).

5. Rich, N. E. et al. Racial and ethnic disparities in nonalcoholic fatty liver disease prevalence, severity, and outcomes in the united states: a systematic review and meta-analysis. Clin. Gastroenterol. Hepatol. 16, 198-210 (2018).

6. Penner, L. A. et al. The effects of oncologist implicit racial bias in racially discordant oncology interactions. J. Clin. Oncol. 34, 2874-2880 (2016).

7. Rubix Life Sciences. COVID-19 and minority health access. https://rubixls.com/wp-content/uploads/2020/04/COVID-19 Minority-Health-Access-7-1.pdf (Rubix, 2020).

8. Dorn, A. V., Cooney, R. E. \& Sabin, M. L. COVID-19 exacerbating inequalities in the US. Lancet 395, 1243-1244 (2020).

9. Williams, D. R. \& Cooper, L. A. COVID-19 and health equity-a new kind of "herd immunity". JAMA https://doi.org/10.1001/ jama.2020.8051 (2020).

10. Issaka, R. \& Somsouk, M. Colorectal cancer screening and prevention in the COVID-19 era. JAMA Health Forum. https:// jamanetwork.com/channels/health-forum/fullarticle/2766137 (2020)

Competing interests

The authors declare no competing interests. 